\title{
Evidence for a Transverse Single-Spin Asymmetry in Leptoproduction of $\pi^{+} \pi^{-}$Pairs
}

\author{
HeRmes Collaboration \\ HERMES - DESY, Notkestraße 85, D-22607 Hamburg
}

\begin{abstract}
A single-spin asymmetry was measured in the azimuthal distribution of $\pi^{+} \pi^{-}$ pairs produced in semi-inclusive deep-inelastic scattering on a transversely polarized hydrogen target. For the first time, evidence is found for a correlation between the transverse target polarization and the azimuthal orientation of the plane containing the two pions. The corresponding single-spin asymmetry is expected to be related to the product of the little-known quark transversity distribution function and an unknown naive-T-odd chiralodd dihadron fragmentation function.
\end{abstract}

KEYWORDS: Lepton-Nucleon Scattering. 
Three fundamental parton distribution functions describe the structure of the nucleon at leading twist: the unpolarized distribution, the helicity distribution, and the transversity distribution. Transversity describes the distribution of transversely polarized quarks in a nucleon with polarization transverse to the direction of the hard probe and is the most difficult one to measure. (For a review see Ref. [何.) Unlike the other two, it is inaccessible in inclusive deep-inelastic scattering (DIS). A class of observables sensitive to the transversity distribution is that of single-spin asymmetries in semi-inclusive DIS on a transversely polarized target.

In general, single-spin asymmetries are related to mixed products of the type $\boldsymbol{S}$. $\left(\boldsymbol{P}_{1} \times \boldsymbol{P}_{2}\right)$, where $\boldsymbol{S}$ is a spin vector (typically the spin of the target or of the quark), and $\boldsymbol{P}_{1}$ and $\boldsymbol{P}_{2}$ are two noncollinear momenta. Single-spin asymmetries are odd under naive time reversal (naive-T-odd), i.e., time reversal without the interchange of initial and final states [2]. Single-spin asymmetries are sensitive to physics at the amplitude level, as they can arise only from the interference between two scattering amplitudes with different phases. Because of the structure of the mixed product, single-spin asymmetries require an interplay between a spin and an orbital angular momentum.

Azimuthal single-spin asymmetries in single-hadron production in semi-inclusive DIS $\left(e p \rightarrow e^{\prime} h X\right)$ on a transversely polarized target were recently measured by the HERMES collaboration for charged pions [3] and by the Compass collaboration for unidentified charged hadrons [4, 5]. For these observables, the orientation of the target transverse polarization influences the distribution of hadrons in the azimuthal angle around the virtual-photon direction through, e.g., the so-called Collins [6] and Sivers [7] mechanisms. In particular, the Collins asymmetry is sensitive to the transversity distribution. At the partonic level, this asymmetry arises from the process in which initially a transversely polarized quark in the transversely polarized target absorbs the virtual photon. The orientation of the transverse polarization of the quark changes in a manner calculable using QED. In the subsequent hadronization of the quark, the direction of the momentum of the detected hadron can be related to the direction of the spin of the quark via the mixed product $\boldsymbol{S}_{q} \cdot\left(\boldsymbol{p}_{q} \times \boldsymbol{P}_{h}\right)$, where $\boldsymbol{p}_{q}$ is the momentum of the struck quark, $\boldsymbol{S}_{q}$ its spin and $\boldsymbol{P}_{h}$ is the momentum of the detected hadron. If such a correlation exists, the hadron has a preference to move to a specific side with respect to the quark spin and the direction of its momentum. The effect vanishes when integrating over the component of the detected hadron's momentum transverse to the momentum of the fragmenting quark. From a formal point of view, despite the complications due to the presence of transverse momentum, factorization proofs [8, 9] allow the interpretation of the Collins asymmetry in terms of a convolution in quark transverse-momentum space of the transversity distribution with a universal naiveT-odd fragmentation function, the Collins function, which can be considered as an analyzer of the fragmenting quark's transverse polarization. This function can be measured in other processes, e.g., in $e^{+} e^{-}$collisions, and can then be used to extract the transversity distribution from the above asymmetries [10]. The only existing data that have been used to isolate transversity are from such measurements of single-spin asymmetries of single hadrons in semi-inclusive DIS.

By the early 1990s it had already been pointed out that single-spin asymmetries in 
semi-inclusive dihadron ${ }^{1}$ production $\left(e p \rightarrow e^{\prime} h_{1} h_{2} X\right)$ on a transversely polarized target could also be sensitive to transversity [11, 12], thereby providing an independent experimental constraint. The underlying mechanism differs from the Collins mechanism in that the transverse spin of the fragmenting quark is transferred to the relative orbital angular momentum of the hadron pair. Consequently, this mechanism does not require transverse momentum of the hadron pair.

Dihadron fragmentation functions were introduced in Ref. [13]. Polarized dihadron fragmentation functions were studied in Refs. [12, 14, 15, 16]. They are related to the concept of jet-handedness [11, 17, as explained in Ref. [18]. The decomposition of the cross section in terms of quark-distribution and dihadron-fragmentation functions was carried out to leading twist (twist-2) in Ref. [19] and to twist-3 in Ref. [20]. Polarized $\rho^{0}$ fragmentation functions [21, 22, 23, 24] are ( $p$-wave) components of dihadron fragmentation functions, as reflected in the angular distribution of the decay products of the $\rho^{0}$ meson.

Little experimental information exists on the multidimensional kinematic dependence of dihadron fragmentation functions. Invariant-mass spectra of hadron pairs were measured in a number of experiments, some of which studied semi-inclusive DIS [25, 26, 27]. Dihadron fragmentation functions have recently been studied in a nuclear environment [28], as they might be relevant to the phenomenon of jet quenching in heavy-ion physics 29]. Vectormeson polarization was analyzed in $e^{+} e^{-}$and $p p$ collisions [23, 30, 31, 32, 33]. However, these data were not interpreted in terms of dihadron fragmentation functions. Finally, studies of longitudinal jet-handedness gave results consistent with zero 34.

Denoting $2 \boldsymbol{R}$ as the difference of the momenta of the two hadrons $h_{1}$ and $h_{2}$, the hadronization of a transversely polarized quark into the hadron pair can depend on the mixed product $\boldsymbol{S}_{q} \cdot\left(\boldsymbol{p}_{q} \times \boldsymbol{R}\right)$. This would imply a preference of $h_{1}$ to go to a specific side with respect to the spin and the momentum direction of the quark, while $h_{2}$ would go to the opposite side. This preference is revealed in the cross section through a dependence on the angle $\phi_{R \perp}$, the azimuthal angle of $\boldsymbol{R}_{\boldsymbol{T}}$, the component of $\boldsymbol{R}$ transverse to $\boldsymbol{P}_{\boldsymbol{h}}$ (see Fig. 1 for the case of $\pi^{+} \pi^{-}$pairs). Here, $\boldsymbol{P}_{\boldsymbol{h}}$ is the sum of the momenta of the two hadrons. Since $\phi_{R \perp}$ is the azimuthal orientation of the relative transverse momentum of the two hadrons, the correlation described above remains present even if the cross section is integrated over the transverse component $\boldsymbol{P}_{\boldsymbol{h} \perp}$ of $\boldsymbol{P}_{\boldsymbol{h}}$. The benefits of integrating over $\boldsymbol{P}_{\boldsymbol{h} \perp}$ are the following: i) issues related to factorization are simpler [34], ii) the evolution equations for the fragmentation functions involved are known [35, 36], iii) distribution and fragmentation functions appear in a simple product instead of a convolution integral over transverse momentum.

This paper reports a measurement of an azimuthal Fourier amplitude of a single-spin asymmetry in semi-inclusive $\pi^{+} \pi^{-}$production on a transversely polarized hydrogen target, resulting in the first evidence of a naive-T-odd chiral-odd dihadron fragmentation function that can provide access to transversity. It is related to the product of the twist-two chiral-odd transversity distribution $h_{1}^{q}$ (also called $\delta q$ ) for quark flavor $q$ and the twisttwo chiral-odd naive-T-odd dihadron fragmentation function $H_{1, q}^{\varangle}$ [20, 37]. ${ }^{2}$ There are no

\footnotetext{
${ }^{1}$ The two hadrons, i.e., $h_{1}$ and $h_{2}$, have to be of different hadron types.

${ }^{2}$ The superscript $\varangle$ indicates that the fragmentation function does not survive integration over the
} 


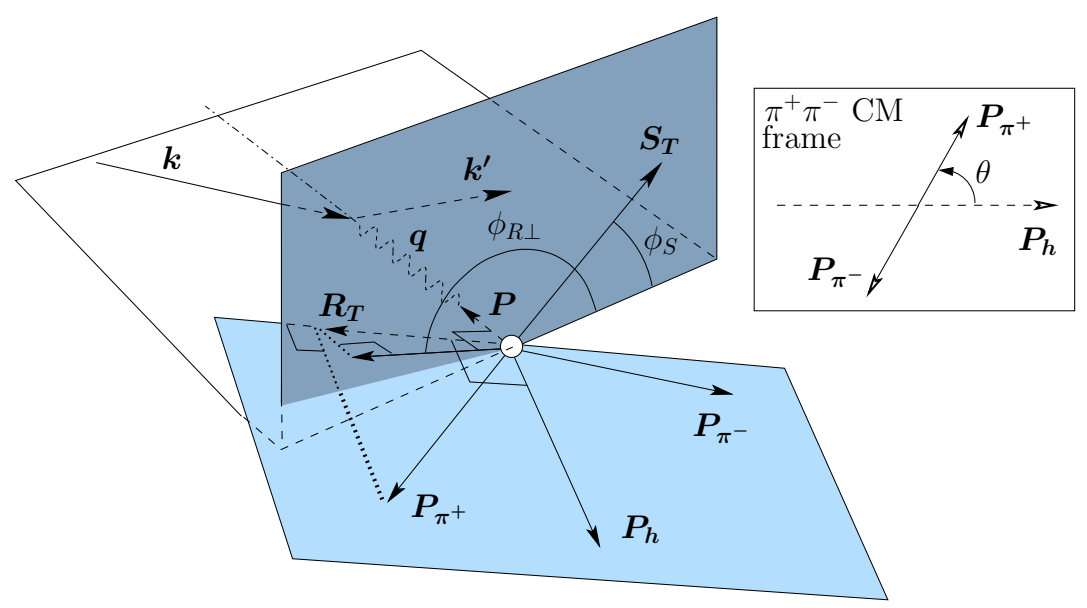

Figure 1: Depiction of the azimuthal angles $\phi_{R \perp}$ of the dihadron and $\phi_{S}$ of the component $\boldsymbol{S}_{\boldsymbol{T}}$ of the target-polarization transverse to both the virtual-photon and target-nucleon momenta $\boldsymbol{q}$ and $\boldsymbol{P}$, respectively. Both angles are evaluated in the virtual-photon-nucleon center-of-momentum frame. Explicitly, $\phi_{R \perp} \equiv \frac{(\boldsymbol{q} \times \boldsymbol{k}) \cdot \boldsymbol{R}_{T}}{\left|(\boldsymbol{q} \times \boldsymbol{k}) \cdot \boldsymbol{R}_{T}\right|} \arccos \frac{(\boldsymbol{q} \times \boldsymbol{k}) \cdot\left(\boldsymbol{q} \times \boldsymbol{R}_{T}\right)}{|\boldsymbol{q} \times \boldsymbol{k}| \boldsymbol{q} \times \boldsymbol{R}_{T} \mid}$ and $\phi_{S} \equiv \frac{(\boldsymbol{q} \times \boldsymbol{k}) \cdot \boldsymbol{S}_{T}}{\left|(\boldsymbol{q} \times \boldsymbol{k}) \cdot \boldsymbol{S}_{T}\right|} \arccos \frac{(\boldsymbol{q} \times \boldsymbol{k}) \cdot\left(\boldsymbol{q} \times \boldsymbol{S}_{\boldsymbol{T}}\right)}{|\boldsymbol{q} \times \boldsymbol{k}|\left|\boldsymbol{q} \times \boldsymbol{S}_{\boldsymbol{T}}\right|}$. Here, $\boldsymbol{R}_{\boldsymbol{T}}=\boldsymbol{R}-\left(\boldsymbol{R} \cdot \hat{\boldsymbol{P}}_{\boldsymbol{h}}\right) \hat{\boldsymbol{P}}_{\boldsymbol{h}}$, with $\boldsymbol{R} \equiv\left(\boldsymbol{P}_{\boldsymbol{\pi}^{+}}-\boldsymbol{P}_{\boldsymbol{\pi}^{-}}\right) / 2, \boldsymbol{P}_{\boldsymbol{h}} \equiv \boldsymbol{P}_{\boldsymbol{\pi}^{+}}+\boldsymbol{P}_{\boldsymbol{\pi}^{-}}$, and $\hat{\boldsymbol{P}}_{\boldsymbol{h}} \equiv \boldsymbol{P}_{\boldsymbol{h}} /\left|\boldsymbol{P}_{\boldsymbol{h}}\right|$, thus $R_{T}$ is the component of $P_{\pi^{+}}$orthogonal to $P_{h}$, and $\phi_{R \perp}$ is the azimuthal angle of $R_{T}$ about the virtual-photon direction. The dotted lines indicate how vectors are projected onto planes. The short dotted line is parallel to the direction of the virtual photon. Also included is a description of the polar angle $\theta$, which is evaluated in the center-of-momentum frame of the pion pair.

contributions to this amplitude at subleading twist (i.e., twist-3). Among the various contributions to the fragmentation function $H_{1, q}^{\varangle}$ are the interference $H_{1, q}^{\varangle, s p}$ between the $s$ - and $p$-wave components of the $\pi^{+} \pi^{-}$pair and the interference $H_{1, q}^{\varangle, p p}$ between two $p$-waves. In some of the literature, such functions have therefore been called interference fragmentation functions [15], even though in general interference between different amplitudes is required by all naive-T-odd functions. In this paper the focus is on the $s p$-interference, since it has received the most theoretical attention. In particular, in Ref. 15 $H_{1, q}^{\varangle, s p}$ was predicted to change sign at a very specific value of the invariant mass $M_{\pi \pi}$ of the $\pi^{+} \pi^{-}$pair, close to the mass of the $\rho^{0}$ meson. However, other models [37, 38] predict a completely different behavior.

The data presented here were recorded during the 2002-2005 running period of the Hermes experiment, using the $27.6 \mathrm{GeV}$ positron or electron beam and a transversely polarized hydrogen gas target internal to the HERA storage ring at DEsY. The openended target cell was fed by an atomic-beam source [39] based on Stern-Gerlach separation combined with transitions of hydrogen hyperfine states. The nuclear polarization of the atoms was flipped at 1-3 min. time intervals, while both this polarization and the atomic fraction inside the target cell were continuously measured 40]. The average value of the transverse proton polarization $\left|S_{\perp}\right|$ was $0.74 \pm 0.06$.

Scattered leptons and coincident hadrons were detected by the HERMES spectrometer [41]. Its acceptance spanned the scattering-angle range $40<\left|\theta_{y}\right|<140 \mathrm{mrad}$ and

relative momentum of the hadron pair. 
$\left|\theta_{x}\right|<170 \mathrm{mrad}$, corresponding to an almost full coverage in $\phi_{S}$ from 0 to $2 \pi$ with only small gaps at $1.40<\phi_{S}<1.74 \mathrm{rad}$ and $4.54<\phi_{S}<4.88 \mathrm{rad}$. Leptons were identified with an efficiency exceeding $98 \%$ and a hadron contamination of less than $1 \%$ using an electromagnetic calorimeter, a transition-radiation detector, a preshower scintillation counter, and a dual-radiator ring-imaging Čerenkov detector [42], mainly used here also to identify charged pions with momentum $\left|\boldsymbol{P}_{\boldsymbol{\pi}}\right|>1 \mathrm{GeV}$.

Events were selected with the kinematic requirements $W^{2}>10 \mathrm{GeV}^{2}, 0.1<y<0.85$, and $Q^{2}>1 \mathrm{GeV}^{2}$, where $W$ is the invariant mass of the initial photon-nucleon system and $y=(P \cdot q) /(P \cdot k)$, with $P, q$, and $k$ representing the four-momenta of the target nucleon, the virtual photon, and the incident lepton, respectively. A constraint was placed on the missing mass: $M_{X}>2 \mathrm{GeV}$. This avoids contributions from exclusive two-pion production, where factorization in distribution and fragmentation functions cannot be applied. All possible combinations of detected $\pi^{+} \pi^{-}$pairs were included for each event, in contrast to keeping only the combination with the largest energy fraction $z$, a choice for which fragmentation functions are not defined. Here, $z$ refers to the fraction of the energy $\nu$ of the virtual photon (in the target rest frame) that is transferred to the pion pair, i.e., $z=\left(E_{\pi^{+}}+E_{\pi^{-}}\right) / \nu=z_{\pi^{+}}+z_{\pi^{-}}$.

In semi-inclusive deep-inelastic scattering of an unpolarized $(U)$ beam off an unpolarized $(U)$ target, the cross section $\sigma_{U U}$ for the production of pion pairs, integrated over the transverse momentum $P_{h \perp}$ of the pion pair, is given, at leading twist and in leading order in $\alpha_{s}\left(\alpha_{s}^{0}\right)$, by 43

$$
\frac{\mathrm{d}^{7} \sigma_{U U}}{\mathrm{~d} x \mathrm{~d} y \mathrm{~d} z \mathrm{~d} \phi_{S} \mathrm{~d} \phi_{R \perp} \mathrm{d} \cos \theta \mathrm{d} M_{\pi \pi}}=\sum_{q} \frac{\alpha^{2} e_{q}^{2}}{2 \pi s x y^{2}}\left(1-y+\frac{y^{2}}{2}\right) f_{1}^{q}(x) D_{1, q}\left(z, M_{\pi \pi}, \cos \theta\right),
$$

where $\alpha$ is the fine-structure constant, $x=Q^{2} /(2 P \cdot q)$, the Mandelstam invariant $s=$ $(P+k)^{2}, f_{1}^{q}$ is the polarization-averaged quark distribution function and $D_{1, q}$ is a dihadron fragmentation function representing the number density of pion pairs produced from unpolarized quarks. The summation runs over the quark and antiquark flavors $q$ with charges $e_{q}$ in units of the elementary charge. For an unpolarized beam and integrating over $P_{h \perp}$, the cross section difference $\sigma_{U T}$ of the polarized cross sections $\sigma_{U \uparrow}$ and $\sigma_{U \downarrow}$, where the target is in either of the two corresponding opposite transverse $(T)$ spin states $\uparrow \downarrow$, is given at leading twist and in leading order in $\alpha_{s}$ by 43

$$
\begin{aligned}
& \frac{\mathrm{d}^{7} \sigma_{U T}}{\mathrm{~d} x \mathrm{~d} y \mathrm{~d} z \mathrm{~d} \phi_{S} \mathrm{~d} \phi_{R \perp} \mathrm{d} \cos \theta \mathrm{d} M_{\pi \pi}} \equiv \frac{1}{2}\left(\mathrm{~d}^{7} \sigma_{U \uparrow}-\mathrm{d}^{7} \sigma_{U \downarrow}\right)= \\
& -\left|\boldsymbol{S}_{\boldsymbol{T}}\right| \sum_{q} \frac{\alpha^{2} e_{q}^{2}}{2 \pi s x y^{2}}(1-y) \frac{1}{2} \sqrt{1-4 \frac{M_{\pi}^{2}}{M_{\pi \pi}^{2}}} \sin \left(\phi_{R \perp}+\phi_{S}\right) \sin \theta h_{1}^{q}(x) H_{1, q}^{\varangle}\left(z, M_{\pi \pi}, \cos \theta\right),
\end{aligned}
$$

where $M_{\pi}$ is the pion mass and $\boldsymbol{S}_{\boldsymbol{T}}$ is the component of the target spin $\boldsymbol{S}$ perpendicular to the virtual-photon direction. The azimuthal angle $\phi_{S}$ always refers to the spin direction,

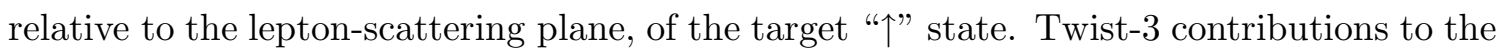
polarized and unpolarized cross sections appear with different azimuthal dependences [20]. 
Both dihadron fragmentation functions $D_{1, q}$ and $H_{1, q}^{\varangle}$ can be expanded in terms of Legendre functions of $\cos \theta$. Hence [43],

$$
D_{1, q}\left(z, M_{\pi \pi}, \cos \theta\right) \simeq D_{1, q}\left(z, M_{\pi \pi}\right)+D_{1, q}^{s p}\left(z, M_{\pi \pi}\right) \cos \theta+D_{1, q}^{p p}\left(z, M_{\pi \pi}\right) \frac{1}{4}\left(3 \cos ^{2} \theta-1\right)
$$

and

$$
H_{1, q}^{\varangle}\left(z, M_{\pi \pi}, \cos \theta\right) \simeq H_{1, q}^{\varangle, s p}\left(z, M_{\pi \pi}\right)+H_{1, q}^{\varangle, p p}\left(z, M_{\pi \pi}\right) \cos \theta,
$$

where the Legendre expansions are truncated to include only the $s$ - and $p$-wave components, which is assumed to be a valid approximation in the range of the invariant mass $M_{\pi \pi}<$ $1.5 \mathrm{GeV}$ [43], which is typical of the present experiment.

In Refs. [15, 37, 43], it was proposed to measure $\sigma_{U U}$ and $\sigma_{U T}$ integrated over the angle $\theta$, which has the advantage that in the resulting expression for these cross sections the only fragmentation functions that appear are $D_{1, q}\left(z, M_{\pi \pi}\right)$ and $H_{1, q}^{\varangle, s p}\left(z, M_{\pi \pi}\right)$ (see Eqs. (1-4)). However, this requires an experimental acceptance that is complete in $\theta$, which is difficult to achieve, not only because of the geometrical acceptance of the detector, but also because of the acceptance in the momentum of the detected pions. As the momentum selection $\left|\boldsymbol{P}_{\boldsymbol{\pi}}\right|>1 \mathrm{GeV}$ strongly influences the $\theta$ distribution, the measured asymmetry must be kept differential in $\theta$.

The single-spin asymmetry $A_{U T} \equiv \frac{1}{\left|\boldsymbol{S}_{\boldsymbol{T}}\right|} \sigma_{U T} / \sigma_{U U}$ contains components of a simultaneous Fourier and Legendre expansion. The amplitude $A_{U T}^{\sin \left(\phi_{R \perp}+\phi_{S}\right) \sin \theta}$ of the modulation of interest here, which is related to the product of transversity and the fragmentation function $H_{1}^{\varangle, s p}$, is defined as

$$
A_{U T}^{\sin \left(\phi_{R \perp}+\phi_{S}\right) \sin \theta} \equiv \frac{2}{\left|\boldsymbol{S}_{\boldsymbol{T}}\right|} \frac{\int \mathrm{d} \cos \theta \mathrm{d} \phi_{R \perp} \mathrm{d} \phi_{S} \sin \left(\phi_{R \perp}+\phi_{S}\right) \mathrm{d} \sigma_{U T}^{7} / \sin \theta}{\int \mathrm{d} \cos \theta \mathrm{d} \phi_{R \perp} \mathrm{d} \phi_{S} \mathrm{~d} \sigma_{U U}^{7}} .
$$

Using Eqs. (114), it can be written as 43

$$
A_{U T}^{\sin \left(\phi_{R \perp}+\phi_{S}\right) \sin \theta}=-\frac{(1-y)}{\left(1-y+\frac{y^{2}}{2}\right)} \frac{1}{2} \sqrt{1-4 \frac{M_{\pi}^{2}}{M_{\pi \pi}^{2}}} \frac{\sum_{q} e_{q}^{2} h_{1}^{q}(x) H_{1, q}^{\varangle, s p}\left(z, M_{\pi \pi}\right)}{\sum_{q} e_{q}^{2} f_{1}^{q}(x) D_{1, q}\left(z, M_{\pi \pi}\right)} .
$$

Due to the factor $e_{q}^{2}$, the amplitude is expected to be up-quark dominated.

The results reported here are extracted from the single-spin asymmetry

$$
A_{U \perp}\left(x, z, M_{\pi \pi}, \phi_{R \perp}, \phi_{S}, \theta\right) \equiv \frac{1}{\left|S_{\perp}\right|} \frac{N^{\uparrow}-N^{\downarrow}}{N^{\uparrow}+N^{\downarrow}},
$$

where $N^{\uparrow(\downarrow)}$ is the luminosity-normalized number of semi-inclusive $\pi^{+} \pi^{-}$pairs detected while the target is in the $\uparrow(\downarrow)$ spin state with polarization perpendicular to the incoming lepton beam (rather than to the virtual-photon direction). The asymmetry is evaluated as a function of $x, z, M_{\pi \pi}$, and the angles $\phi_{R \perp}, \phi_{S}$, and $\theta$, which are defined in Fig. 1. ${ }^{3}$

A $\chi^{2}$ fit was performed, binned in $\left(\phi_{R \perp}+\phi_{S}\right)$ versus $\theta^{\prime} \equiv|| \theta-\pi / 2|-\pi / 2|$, with a function of the form:

$$
A_{U \perp}\left(\phi_{R \perp}+\phi_{S}, \theta^{\prime}\right)=\sin \left(\phi_{R \perp}+\phi_{S}\right) \frac{a \sin \theta^{\prime}}{1+b \frac{1}{4}\left(3 \cos ^{2} \theta^{\prime}-1\right)},
$$

\footnotetext{
${ }^{3}$ The definitions of the asymmetry and the angles are consistent with the "Trento Conventions" 44 .
} 
where $a \equiv A_{U \perp}^{\sin \left(\phi_{R \perp}+\phi_{S}\right) \sin \theta}$ is a free parameter of the fit, while $b$ is varied to study the influence of the unknown contribution $D_{1, q}^{p p}$ to the polarization-averaged 2-hadron cross section. The fit is evaluated as a function of $\theta^{\prime}$, which corresponds to a symmetrization of the fit around $\theta=\pi / 2$. This has the advantage that the contributions to $A_{U \perp}$ containing $D_{1, q}^{s p}$ and $H_{1, q}^{\varangle, p p}$ drop out (see, e.g., Eqs. (3) and (目)), reducing the statistical uncertainty on $a \equiv A_{U \perp}^{\sin \left(\phi_{R \perp}+\phi_{S}\right) \sin \theta}$, the modulation amplitude of interest that approximates $A_{U T}^{\sin \left(\phi_{R \perp}+\phi_{S}\right) \sin \theta}$ defined in Eq. (5).

The value of the fit parameter $a$ depends on the value of $b$. Therefore, a systematic uncertainty was assigned to the extracted value of $a$ by studying its response to variation of $b$. The parameter $b$ was varied within its positivity limits, given by [43]

$$
-\frac{3 D_{1, q}^{p}\left(z, M_{\pi \pi}\right)}{2 D_{1, q}\left(z, M_{\pi \pi}\right)} \leq b \leq \frac{3 D_{1, q}^{p}\left(z, M_{\pi \pi}\right)}{D_{1, q}\left(z, M_{\pi \pi}\right)}
$$

where $D_{1, q}^{p}\left(z, M_{\pi \pi}\right)$ indicates the pure $p$-wave component of the fragmentation functions $D_{1, q}\left(z, M_{\pi \pi}\right)$. The size of this component was estimated using the PYTHIA6 event generator [45] tuned to Hermes data [46]. The strange contribution was neglected, while isospin and charge-conjugation symmetry implies that both $D_{1, q}^{p}\left(z, M_{\pi \pi}\right)$ as well as $D_{1, q}\left(z, M_{\pi \pi}\right)$ have identical values for $q=u, \bar{u}, d, \bar{d}$. Varying the Pyтнia 6 estimate by $20 \%$ does not significantly change the systematic uncertainty assigned to $a$. The presented values for $a$ are the central values in the ranges of $a$ obtained by varying $b$ between its lower and upper bounds, while the "b-scan" uncertainty is taken to be the standard deviation.

The values of the amplitudes $A_{U \perp}^{\sin \left(\phi_{R \perp}+\phi_{S}\right) \sin \theta}$ extracted as functions of $M_{\pi \pi}, x$, and $z$, are shown in Fig. 2 and reported in Table 1. They are positive over the entire range of all three variables. The reduced- $\chi^{2}$ values for the fits to the data set are in the range 0.64-1.38. The measured asymmetry is based on events integrated over $\boldsymbol{P}_{\boldsymbol{h} \perp}$ (within the acceptance), which considerably simplifies an eventual extraction of $h_{1}^{q}$ and $H_{1, q}^{\varangle, s p}$, since in this case $h_{1}^{q} H_{1, q}^{\varangle, s p}$ appears in the expression for the modulation amplitudes as a simple product (see Eq. (6) ) instead of in a convolution integral over transverse momentum.

The value of $A_{U \perp}^{\sin \left(\phi_{R \perp}+\phi_{S}\right) \sin \theta}$ extracted from events summed over the experimental acceptance is $A_{U \perp}^{\sin \left(\phi_{R \perp}+\phi_{S}\right) \sin \theta}=0.018 \pm 0.005_{\text {stat }} \pm 0.002_{\mathrm{b}-\text { scan }}$, with an additional $8.1 \%$ scale uncertainty coming from the uncertainty in the determination of the target polarization. As discussed below, acceptance effects were found to lead to an underestimate of the true value of the modulation amplitude by up to $20 \%$. For this result, the ranges selected in $x$ and $M_{\pi \pi}$ are $0.023<x<0.4$ and $0.5 \mathrm{GeV}<M_{\pi \pi}<1.0 \mathrm{GeV}$. The mean values of the kinematic variables are $\langle x\rangle=0.07,\langle y\rangle=0.64,\left\langle Q^{2}\right\rangle=2.35 \mathrm{GeV}^{2},\langle z\rangle=0.43$, and $\left\langle\left|\boldsymbol{P}_{\boldsymbol{h} \perp}\right|\right\rangle=0.42 \mathrm{GeV}$.

The modulation amplitudes extracted are not influenced by the addition in the fit of terms of the form $\sin \phi_{S}$ (which appears at subleading twist in the polarized cross section $\sigma_{U T}$ ), or of the form $\cos \phi_{R \perp} \sin \theta$ (which appears at subleading twist in the unpolarized cross section $\left.\sigma_{U U}\right)$. These angular combinations exhaust the possibilities up to subleading twist. In order to eliminate effects of the natural polarization of the HERA lepton beam, data with both beam-helicity states were combined. The resulting net beam polarization is 


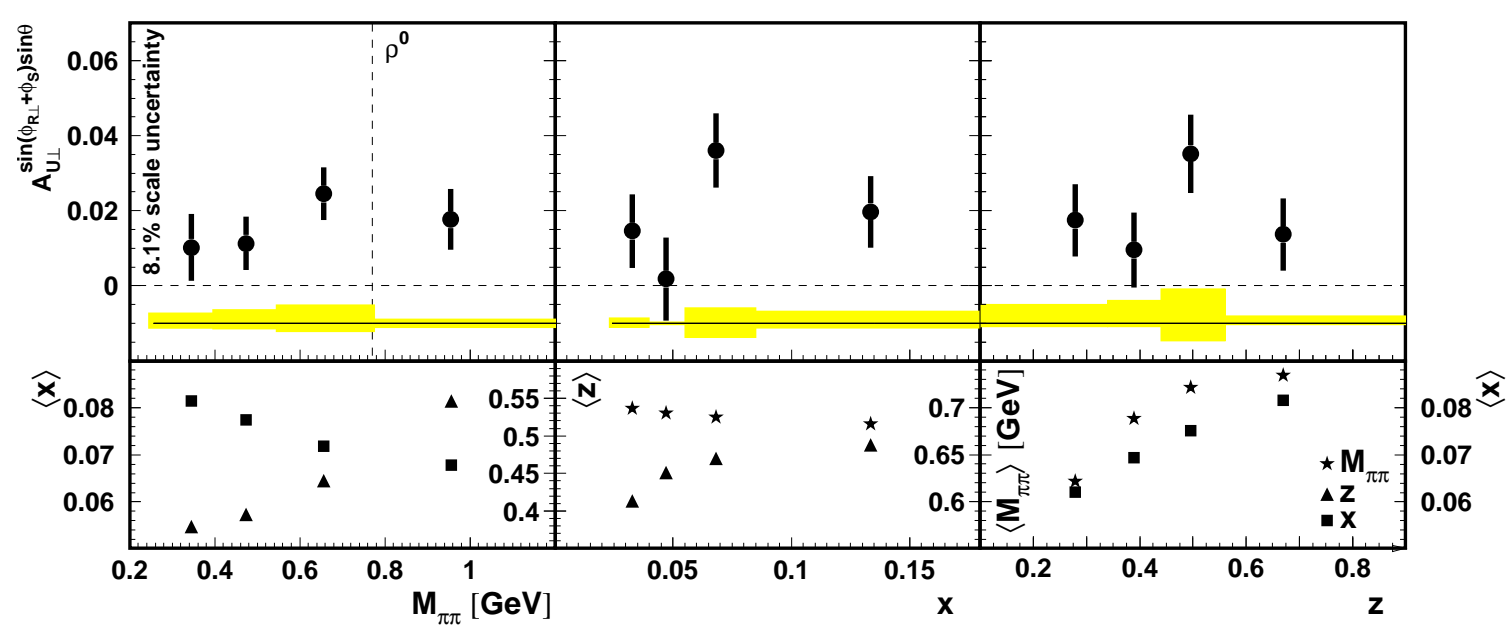

Figure 2: The top panels show $A_{U \perp}^{\sin \left(\phi_{R \perp}+\phi_{S}\right) \sin \theta}$ versus $M_{\pi \pi}, x$, and $z$. The bottom panels show the average values of the variables that were integrated over. For the dependence on $x$ and $z$, $M_{\pi \pi}$ was constrained to the range $0.5 \mathrm{GeV}<M_{\pi \pi}<1.0 \mathrm{GeV}$, where the signal is expected to be largest. The error bars show the statistical uncertainty. A scale uncertainty of $8.1 \%$ arises from the uncertainty in the target polarization. Other contributions to the systematic uncertainty are summed in quadrature and represented by the asymmetric error band.

$-0.020 \pm 0.001$. The influence of this small but nonzero net polarization on the amplitude extracted was shown to be negligible by analyzing separately the data of the two beamhelicity states. There is also no influence from the addition to the fit of a constant term, the latter being consistent with zero. Identical results were obtained using an unbinned maximum-likelihood fit.

Tracking corrections that are applied for the deflections of the scattered particles caused by the vertical $0.3 \mathrm{~T}$ target holding field have also a negligible effect on the extracted asymmetries.

The fully differential asymmetry depends on nine kinematic variables: $x, y, z, \phi_{R \perp}$, $\phi_{S}$, and $\theta, M_{\pi \pi}$, and $\boldsymbol{P}_{\boldsymbol{h} \perp}\left(\mathrm{d}^{2} \boldsymbol{P}_{\boldsymbol{h} \perp}=\left|\boldsymbol{P}_{\boldsymbol{h} \perp}\right| \mathrm{d}\left|\boldsymbol{P}_{\boldsymbol{h} \perp}\right| \mathrm{d} \phi_{h}\right)$. Due to the limited statistical precision, it is not possible to measure the asymmetry $A_{U \perp}$ fully differential in all relevant variables. Combined with the fact that the HeRmes spectrometer does not have a full $4 \pi$ acceptance, this implies that the measured number of events is always convolved with the experimental acceptance $\epsilon$, e.g.,

$$
\begin{aligned}
N^{\uparrow(\downarrow)}\left(\phi_{R \perp}, \phi_{S}, \theta, M_{\pi \pi}\right) \propto & \int \mathrm{d} x \mathrm{~d} y \mathrm{~d} z \mathrm{~d}^{2} \boldsymbol{P}_{\boldsymbol{h} \perp} \epsilon\left(x, y, z, \boldsymbol{P}_{\boldsymbol{h} \perp}, \phi_{R \perp}, \phi_{S}, \theta, M_{\pi \pi}\right) \times \\
& \times \sigma_{U \uparrow(\downarrow)}\left(x, y, z, \boldsymbol{P}_{\boldsymbol{h} \perp}, \phi_{R \perp}, \phi_{S}, \theta, M_{\pi \pi}\right),
\end{aligned}
$$

such that $\epsilon$ does not necessarily drop out of the expression for the asymmetry (Eq. (7) $)^{4}$. Some effects of the acceptance can be easily dealt with if the predicted asymmetry amplitude is linearly dependent on all variables in the range over which they are integrated.

\footnotetext{
${ }^{4}$ Note that, experimentally, the asymmetry itself is never integrated directly over any variables: always the numerator and denominator of the asymmetry are integrated separately.
} 
In that case, the measured amplitudes are equal to the true amplitudes evaluated at the average values of these variables. However, all models predict a highly nonlinear behavior of the amplitude as a function of the invariant mass $M_{\pi \pi}$. Moreover, when the integration of the cross section over $\boldsymbol{P}_{\boldsymbol{h} \perp}$ is incomplete because of the geometrical acceptance, other

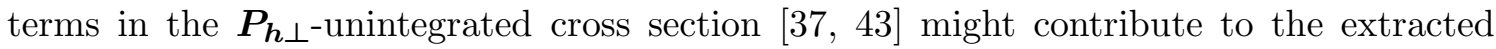
amplitudes.

Therefore, a systematic uncertainty was estimated based on a Monte Carlo study, which is explained in more detail in the Appendix. In particular, two possible sources of systematic uncertainties have been examined: the difference in the modulation amplitude of interest extracted as done for real data in the experimental acceptance and similarly in $4 \pi$ acceptance, and a possible false asymmetry originating from other terms appearing through incomplete integration over $\boldsymbol{P}_{\boldsymbol{h} \perp}$.

The largest effect was seen when comparing the amplitudes in $4 \pi$ and in the experimental acceptance. The Monte Carlo simulation used a particular choice for transversity and for each of the dihadron fragmentation functions, which results in a reasonable description of the kinematic dependences of the measured amplitudes (cf. Figs. 1 and 2). The amplitudes extracted in the experimental acceptance were found to be underestimated by up to $43 \%$ for certain values of $z$ when compared to amplitudes extracted in $4 \pi$ coverage. The effect was negligible for all $x$ bins when integrating over $z$, and about $21 \%$ when integrated over the whole kinematic range. No other models for the dihadron functions involved, suitable for this simulation, are presently available. This systematic uncertainty estimate applies only when interpreting the results as values based on separate integration of numerator and denominator of the asymmetry over the relevant ranges of all kinematic variables. This choice was necessitated by the strong model-dependence of the acceptance effects when not integrating over $M_{\pi \pi}$.

The incomplete integration over $\boldsymbol{P}_{\boldsymbol{h} \perp}$ was found to have only a small influence on the

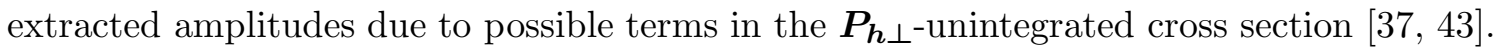
In view of the large uncertainties above, it can be neglected.

The interpretation of the amplitudes extracted can, in principle, be complicated by the experimental condition that the target polarization is transverse to the beam axis instead of transverse to the virtual-photon direction. These beam-axis asymmetries can receive contributions not only from the transverse component of the nucleon spin with respect to the virtual-photon direction but also from a small longitudinal component proportional to $\sin \theta_{\gamma^{*}}$, where $\theta_{\gamma^{*}}$ is the angle between the directions of the virtual photon and the incoming lepton beam. Such a contribution to the amplitude presented here can occur only when a $\sin \phi_{R \perp}$ amplitude exists in the corresponding asymmetry $A_{U L}$, i.e., the photon-axis asymmetry in dihadron lepto-production with an unpolarized beam on a longitudinally polarized target [47]. Such an amplitude exists at subleading twist [20], but was measured to be small for pairs of unidentified hadrons 48. In addition, $\left\langle\sin \theta_{\gamma^{*}}\right\rangle$ is typically less than 0.09 [48], leading to an insignificant difference in the presented amplitude for lepton-axis and photon-axis asymmetries.

Besides this contribution, no other twist-3 effects are present in the measured amplitude. Modifications due to even higher twist and NLO effects are unknown for dihadron 


\begin{tabular}{c|c|c}
\hline \hline bin boundaries & $A_{U \perp}^{\sin \left(\phi_{R \perp}+\phi_{S}\right) \sin \theta}$ & reduced $\chi^{2}$ \\
\hline $0.25 \mathrm{GeV}<M_{\pi \pi}<0.40 \mathrm{GeV}$ & $0.010 \pm 0.009_{\text {stat }} \pm 0.001_{\mathrm{b}-\text { scan }}+0.002_{\mathrm{acc}}$ & 0.70 \\
$0.40 \mathrm{GeV}<M_{\pi \pi}<0.55 \mathrm{GeV}$ & $0.012 \pm 0.007_{\text {stat }} \pm 0.001_{\mathrm{b}-\text { scan }}+0.003_{\mathrm{acc}}$ & 1.32 \\
$0.55 \mathrm{GeV}<M_{\pi \pi}<0.77 \mathrm{GeV}$ & $0.024 \pm 0.007_{\text {stat }} \pm 0.002_{\mathrm{b}-\text { scan }}+0.004_{\mathrm{acc}}$ & 0.85 \\
$0.77 \mathrm{GeV}<M_{\pi \pi}<2.00 \mathrm{GeV}$ & $0.019 \pm 0.008_{\text {stat }} \pm 0.001_{\mathrm{b}-\text { scan }}+0.000_{\mathrm{acc}}$ & 0.96 \\
\hline $0.023<x<0.040$ & $0.015 \pm 0.010_{\text {stat }} \pm 0.001_{\mathrm{b}-\text { scan }}+0.001_{\mathrm{acc}}$ & 0.88 \\
$0.040<x<0.055$ & $0.002 \pm 0.011_{\text {stat }} \pm 0.001_{\mathrm{b}-\text { scan }}+0.000_{\mathrm{acc}}$ & 1.03 \\
$0.055<x<0.085$ & $0.035 \pm 0.010_{\text {stat }} \pm 0.004_{\mathrm{b}-\text { scan }}+0.002_{\mathrm{acc}}$ & 1.38 \\
$0.085<x<0.400$ & $0.020 \pm 0.010_{\text {stat }} \pm 0.001_{\mathrm{b}-\text { scan }}+0.003_{\mathrm{acc}}$ & 0.94 \\
\hline $0.000<z<0.340$ & $0.018 \pm 0.010_{\text {stat }} \pm 0.001_{\mathrm{b}-\text { scan }}+0.005_{\mathrm{acc}}$ & 1.04 \\
$0.340<z<0.440$ & $0.010 \pm 0.010_{\text {stat }} \pm 0.001_{\mathrm{b}-\text { scan }}+0.006_{\mathrm{acc}}$ & 0.64 \\
$0.440<z<0.560$ & $0.036 \pm 0.010_{\text {stat }} \pm 0.005_{\mathrm{b}-\text { scan }}+0.008_{\mathrm{acc}}$ & 1.04 \\
$0.560<z<1.000$ & $0.012 \pm 0.009_{\text {stat }} \pm 0.001_{\mathrm{b}-\text { scan }}+0.002_{\mathrm{acc}}$ & 0.84 \\
\hline $0.5 \mathrm{GeV}<M_{\pi \pi}<1.0 \mathrm{GeV}$ & & \\
$0.023<x<0.400$ & $0.018 \pm 0.005_{\text {stat }} \pm 0.002_{\mathrm{b}-\text { scan }}+0.004_{\mathrm{acc}}$ & 0.87 \\
$0.0<z<1.0$ & & \\
\hline \hline
\end{tabular}

Table 1: The extracted modulation amplitudes with statistical uncertainty, the systematic uncertainties arising from the scan of $b$ in the fits and from extracting the amplitudes in the experimental acceptance as described in the text. A further $8.1 \%$ scale uncertainty from the target polarization is not listed. In addition, the bin boundaries are given in the various $M_{\pi \pi^{-}}, x_{-}$, and $z$-bins, respectively, as well as the reduced- $\chi^{2}$ values of the fits. Note that for both the $x$ and $z$ dependences, the lower and upper limits on $M_{\pi \pi}$ are $0.5 \mathrm{GeV}$ and $1 \mathrm{GeV}$, respectively. The acceptance effect in the last row is not an average over those values for $x$ or $z$ bins because the bin weighting for the amplitudes in $4 \pi$ differs from those for experimental acceptance.

production in DIS. However, the dominant NLO contribution to the "longitudinal" cross section $\sigma_{L}$ is known to be up to $30 \%$ for the unpolarized inclusive DIS cross section in these kinematic conditions 49].

Since the fragmentation functions $H_{1, q}^{\varangle, s p}$ require the interference between $s$ and $p$ waves, it is supposed to be sizeable in the regions where spin- 1 resonances are present, assuming the rest of the spectrum to be in an $s$ wave. As can be seen in Fig. 3, in the invariant-mass range explored in this paper the $\rho^{0}$ and $\omega$ resonances are present and give large contributions to the spectrum. The available theoretical models indicate that $H_{1, q}^{\varangle, s p}$ should be maximal in the vicinity of the $\rho^{0}$ mass [15, 37, 38].

Being naive-T-odd, the fragmentation function requires the interference between scattering amplitudes with different phases. The model of Ref. [15] considers the interference between the $\rho^{0}$ and the $\sigma$ resonance, as measured in $\pi^{+} \pi^{-}$scattering, predicting a sign change of the fragmentation function close to the $\rho^{0}$ mass. The models of Refs. [37, 38] neglect the contributions from the $\sigma$ resonance and assume the $s$-wave amplitude of the spectrum to be real. Thus, the fragmentation function turns out to be almost proportional to the imaginary part of the $\rho^{0}$ resonance, i.e., a Breit-Wigner shape peaked at the $\rho^{0}$ 




Figure 3: Yield distribution in the invariant mass of the $\pi^{+} \pi^{-}$pairs for the experimental data compared to a Pyтhia6 Monte Carlo simulation. Both distributions are normalized to unity. The main resonances contributing to the simulated spectrum are shown separately.

mass. In Ref. [38], the imaginary part of the $\omega$ resonance is also taken into account, giving rise to an additional contribution to the fragmentation function in the region around $M_{\pi \pi} \approx 0.5 \mathrm{GeV}$.

The $M_{\pi \pi}$ dependence of the measured modulation amplitude shows no sign change at the $\rho^{0}$ mass, contrary to the prediction in Ref. [15]. This leads to the conclusion that $\rho-\sigma$ interference is not the dominant contribution to the fragmentation function $H_{1}^{\varangle, s p}$, and that in general interference patterns observed in semi-inclusive $\pi^{+} \pi^{-}$production are different from those observed in $\pi^{+} \pi^{-}$scattering. The dependences on $M_{\pi \pi}$ and $z$ of the model calculations of Ref. [38] (see also [50]), one of which is reproduced in Fig. \&, are not inconsistent in shape with the present data. However, the predictions are at least a factor of two larger.

In summary, a measurement of $A_{U \perp}^{\sin \left(\phi_{R \perp}+\phi_{S}\right) \sin \theta}$ of the transverse-target-spin asymmetry in the lepto-production of $\pi^{+} \pi^{-}$pairs has provided the first evidence that a naive-T-odd chiral-odd dihadron fragmentation function $H_{1, q}^{\varangle}$ and in particular $H_{1, q}^{\varangle, s p}$ is nonzero. The average value of the amplitude is $A_{U \perp}^{\sin \left(\phi_{R \perp}+\phi_{S}\right) \sin \theta}=0.018 \pm 0.005_{\text {stat }} \pm 0.002_{\mathrm{b}-\text { scan }}+0.004_{\text {acc }}$, with an additional $8.1 \%$ scale uncertainty. The amplitude is positive in the whole range in the invariant mass of the $\pi^{+} \pi^{-}$pairs, in contrast to a previous expectation [15] of a sign change around the mass of the $\rho^{0}$ meson. Possibly the most striking aspect of the reported results is the relatively large size of an asymmetry caused by a complicated interference effect.

A mechanism analogous to the one investigated in this paper offers perhaps the most promising way to access transversity in $p p$ collisions at RHIC. Our results show for the first time that this mechanism can indeed give a sizeable signal. The Belle collaboration can extract dihadron fragmentation functions from their $e^{+} e^{-}$data. Such results could then be combined with DIS and $p p$ data to extract transversity in the proton. 


\section{Acknowledgments}

We gratefully acknowledge the DESY management for its support and the staff at DESY and the collaborating institutions for their significant effort. This work was supported by the FWO-Flanders and IWT, Belgium; the Natural Sciences and Engineering Research Council of Canada; the National Natural Science Foundation of China; the Alexander von Humboldt Stiftung; the German Bundesministerium für Bildung und Forschung (BMBF); the Deutsche Forschungsgemeinschaft (DFG); the Italian Istituto Nazionale di Fisica Nucleare (INFN); the MEXT, JSPS, and COE21 of Japan; the Dutch Foundation for Fundamenteel Onderzoek der Materie (FOM); the U. K. Engineering and Physical Sciences Research Council, the Particle Physics and Astronomy Research Council and the Scottish Universities Physics Alliance; the U. S. Department of Energy (DOE) and the National Science Foundation (NSF); the Russian Academy of Science and the Russian Federal Agency for Science and Innovations; the Ministry of Trade and Economical Development and the Ministry of Education and Science of Armenia; and the European Community-Research Infrastructure Activity under the FP6 "Structuring the European Research Area" program (Hadron Physics, contract number RII3-CT-2004-506078).

\section{A. Description of the Monte Carlo Study}

The starting point of the acceptance studies was a Pyтнia6 Monte Carlo simulation 445, which does not have any processes related to transverse target polarization. Specifically, a version of Pythia6 was used where the relevant cross sections were tuned to HeRMES data 46]. The target-polarization dependence was introduced by randomly assigning spin states to events with a probability according to an expression for $A_{U T}$ as a function of the various kinematic variables.

In the first study, only the modulation amplitude of interest was implemented in order to assess the effects of the acceptance on it. For the dihadron fragmentation functions $D_{1, q}\left(z, M_{\pi \pi}\right)$ and $H_{1, q}^{\varangle, s p}\left(z, M_{\pi \pi}\right)$, the models of Ref. [38] were implemented. For the distribution functions $f_{1}^{q}(x)$ and $h_{1}^{q}(x)$, parameterizations were taken from Ref. [51] and from Ref. [52], respectively. No additional dependence on transverse momentum was introduced, i.e., it was assumed that any dependence on transverse momentum of the products of polarized and unpolarized distribution and fragmentation functions cancels in the asymmetry.

Modulation amplitudes were then extracted in a fit ${ }^{5}$ to both the data in $4 \pi$ and the HERMES experimental acceptance, where the latter was simulated with a parameterization of the spectrometer performance based on GEANT3. The shape of the yield distributions in all nine kinematic variables in the experimental acceptance can be found in Ref. 553. As shown in Fig. 4 , the acceptance effect can be quite large: the modulation amplitudes extracted in the experimental acceptance are underestimated by up to $25 \%$ in certain $M_{\pi \pi}$ bins and by up to $43 \%$ for certain $z$ bins when compared to amplitudes extracted in $4 \pi$ coverage. The effect was negligible in all $x$ bins. Apparent is the discrepancy in the

\footnotetext{
${ }^{5}$ For this study it was assumed that the acceptance in $\theta$ is complete, i.e., no contribution from $b$ was taken into account in Eq. (8).
} 
average values of $x$ for $4 \pi$ and the experimental acceptances, where a strong dependence of the asymmetry on $x$, which is driven by the increase of transversity with $x$ in the range considered, leads to the observed underestimates in the amplitudes extracted when integrated over $x$.

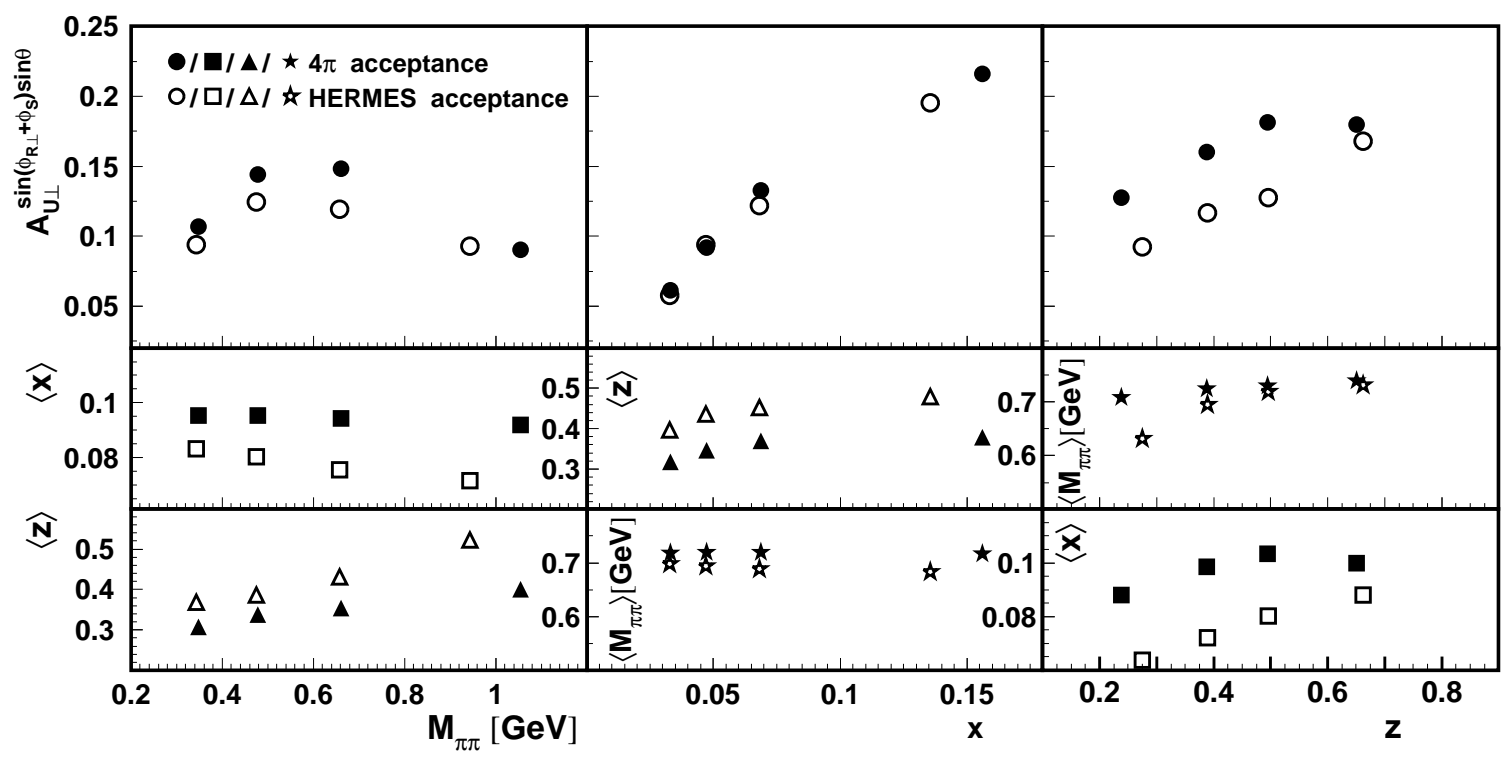

Figure 4: The top panels show $A_{U \perp}^{\sin \left(\phi_{R \perp}+\phi_{S}\right) \sin \theta}$ versus $M_{\pi \pi}, x$, and $z$ for Monte-Carlo data extracted both in $4 \pi$ and experimental acceptance. The bottom panels show the average values of the variables that were integrated over. For the dependence on $x$ and $z, M_{\pi \pi}$ was constrained to the range $0.5 \mathrm{GeV}<M_{\pi \pi}<1.0 \mathrm{GeV}$. The systematic uncertainties assigned to the amplitudes extracted from real data (listed in Table 1) are obtained from the differences between the above amplitudes in the experimental acceptance as compared to $4 \pi$. These differences were scaled by the ratio of the average reconstructed amplitudes obtained from HERMES data and from the Monte Carlo data in order to accommodate the larger magnitude of the model prediction.

A second study dealt with contributions from contaminating modulation amplitudes appearing through the incomplete integration over $\boldsymbol{P}_{\boldsymbol{h} \perp}$. The experimental acceptance has a strong dependence on $\phi_{h}$, the azimuthal angle of $P_{h}$ around the virtual-photon direction, with the consequence that the extracted amplitude $a$ in Eq. (8) does not necessarily correspond to Eq. (6). The fully differential $\phi_{h}$-dependent cross section [37, 43] contains many terms, which if nonzero and if the integral over $\phi_{h}$ is incomplete, can give unwanted contributions to the modulation amplitude.

In principle, these terms could be taken into account in the fit (Eq. (8)), but this is difficult with the current statistical precision of this measurement, as it would require, e.g., 3-dimensional binning, i.e., an additional binning in $\phi_{h}$. To study the influence on the amplitude of interest, model predictions for the size and dependences of all these $\phi_{h}$-dependent terms are necessary. However, no such information exists, i.e., most of the distribution and fragmentation functions involved are as yet completely unknown. In order to estimate a systematic uncertainty, a very general model was used for these terms, 
varying their size and dependences. The averages of the effect on the extracted value of $A_{U \perp}^{\sin \left(\phi_{R \perp}+\phi_{S}\right) \sin \theta}$ were then used to estimate a contribution to the systematic uncertainty.

Target spin states were again assigned to semi-inclusive events from a PyTHIA6 Monte Carlo simulation according to a model for the asymmetry $A_{U T}$, but now including all $\phi_{h^{-}}$ dependent terms. For the distribution functions $f_{1}^{q}(x), h_{1}^{q}(x)$ and for the fragmentation functions $D_{1, q}\left(M_{\pi \pi}, z\right)$ and $H_{1, q}^{\varangle, s p}\left(M_{\pi \pi}, z\right)$ the same models were used as before. For the transverse-momentum dependence of all distribution and fragmentation functions appearing in $A_{U T}$, a Gaussian Ansatz was used:

$$
\begin{gathered}
f_{1}\left(x, \boldsymbol{p}_{\boldsymbol{T}}^{2}\right)=\frac{1}{\pi\left\langle\boldsymbol{p}_{\boldsymbol{T}}^{2}\right\rangle} e^{-\frac{p_{\boldsymbol{T}}^{2}}{\left\langle\boldsymbol{p}_{\boldsymbol{T}}^{2}\right\rangle}} f_{1}(x), \\
D_{1}\left(z, M_{\pi \pi}, \cos \theta, \boldsymbol{k}_{\boldsymbol{T}}^{2}, \boldsymbol{k}_{\boldsymbol{T}} \cdot \boldsymbol{R}_{\boldsymbol{T}}\right)=\frac{1}{z^{2} \pi\left\langle\boldsymbol{k}_{\boldsymbol{T}}^{2}\right\rangle} e^{-\frac{\boldsymbol{k}_{T}^{2}}{\left\langle\boldsymbol{k}_{\boldsymbol{T}}^{2}\right\rangle} D_{1}\left(z, M_{\pi \pi}, \cos \theta\right) .}
\end{gathered}
$$

with $\boldsymbol{p}_{\boldsymbol{T}}\left(\boldsymbol{k}_{\boldsymbol{T}}\right)$ being the initial- (final-/fragmenting-) quark's momentum component that is transverse to the initial- (final-) hadron's momentum direction. The same $\boldsymbol{p}_{\boldsymbol{T}}^{2}$ and $\boldsymbol{k}_{\boldsymbol{T}}^{2}$ dependences were used for all other distribution and fragmentation functions. The actual values of $\boldsymbol{p}_{\boldsymbol{T}}^{2}$ and $\boldsymbol{k}_{\boldsymbol{T}}^{2}$ are irrelevant as they are absorbed in the $C_{N}$ in Eq. A.3.

The $\phi_{h}$-dependent terms were implemented such that the corresponding azimuthal amplitudes $A_{U T}^{\sin \left(a \phi_{h}+b \phi_{R \perp}+c \phi_{S}+\frac{d}{2} \pi\right)}$ depend on $x, z$, and $\boldsymbol{P}_{\boldsymbol{h} \perp}$ according to

$$
\begin{aligned}
\frac{1}{2} A_{U T}^{\sin \left(a \phi_{h}+b \phi_{R \perp}+c \phi_{S}+\frac{d}{2} \pi\right)} & \equiv \frac{\int \mathrm{d} \phi_{h} \mathrm{~d} \phi_{R \perp} \mathrm{d} \phi_{S} \sin \left(a \phi_{h}+b \phi_{R \perp}+c \phi_{S}+\frac{d}{2} \pi\right) \mathrm{d}^{9} \sigma_{U T}}{\int \mathrm{d} \phi_{h} \mathrm{~d} \phi_{R \perp} \mathrm{d} \phi_{S} \mathrm{~d}^{9} \sigma_{U U}} \\
& =C_{N} z^{\alpha_{N}} x^{\beta_{N}} f_{N}\left(\left|\boldsymbol{P}_{\boldsymbol{h} \perp}\right|\right)
\end{aligned}
$$

with $N$ identifying the various possible terms in the full polarized cross section [37, 43], $C_{N}$ a constant scaling factor, $\alpha_{N}, \beta_{N} \in[0.1,3]$ and $a, b, c$ and $d$ are either zero or integers depending on $N$. The interval $[0.1,3]$ is based on typical parameterizations of the parton distributions $f_{1}^{q}$ and the single-hadron fragmentation function $D_{1, q}(z)$. Similarly, azimuthal amplitudes $A_{U U}^{\sin \left(a \phi_{h}+b \phi_{R \perp}+c \phi_{S}+\frac{d}{2} \pi\right)}$ were introduced for the $\phi_{h}$-dependent parts in the unpolarized cross section. Apart from the fact that all these different modulation amplitudes of the polarized and unpolarized cross section increase nonlinearly with increasing $x$ and $z$, the choices for $\alpha_{N}$ and $\beta_{N}$ are quite arbitrary, but were found not to influence the final conclusions. Starting from the expressions for the convolution integrals in the involved cross sections [37, 43] and using the Gaussian Ansätze Eqs. (A.1, A.2) for the $\boldsymbol{p}_{\boldsymbol{T}}^{2}$ and $\boldsymbol{k}_{\boldsymbol{T}}^{2}$ dependence of the distribution and fragmentation functions, the dependences $f_{N}\left(\left|\boldsymbol{P}_{\boldsymbol{h} \perp}\right|\right)$ of the modulation amplitudes on $\left|\boldsymbol{P}_{\boldsymbol{h} \perp}\right|$ were derived [53]. ${ }^{6}$

The values of the scaling factors $C_{N}$ in Eq. A.3 were derived from the averaged modulation amplitudes, which were randomly chosen in the range $[-0.1,0.1]$, i.e.,

$$
\frac{\int A_{U U / T}^{\sin \left(a \phi_{h}+b \phi_{R \perp}+c \phi_{S}+\frac{d}{2} \pi\right)} \mathrm{d}^{9} \sigma_{U U}}{\int \mathrm{d}^{9} \sigma_{U U}} \in[-0.1,0.1],
$$

\footnotetext{
${ }^{6}$ Due to the fact that no $\boldsymbol{k}_{\boldsymbol{T}} \cdot \boldsymbol{R}_{\boldsymbol{T}}$ dependence is taken into account for the fragmentation functions, about half of the $\phi_{h}$-dependent terms drop out of the complete expression for the polarized cross section.
} 
where the integral is performed over all nine dimensions and integration ranges were used corresponding to those used in the analysis. Each resulting parameterization of $A_{U T}$ had to satisfy the positivity limit $\left|A_{U T}\right|<1$.

To estimate the systematic uncertainty, the amplitude $A_{U \perp}^{\sin \left(\phi_{R \perp}+\phi_{S}\right) \sin \theta}$ was extracted 1000 times from the same Pythia6 dataset, similar in size to the real data, but each time with spin states randomly chosen according to their probability calculated from randomly chosen values for $\alpha_{N}, \beta_{N}$, and $C_{N}$ for each of the $\phi_{h}$-dependent terms. The distribution obtained in the extracted amplitudes $A_{U \perp}^{\sin \left(\phi_{R \perp}+\phi_{S}\right) \sin \theta}$ was compared to a similarly obtained distribution, but which had only $A_{U T}^{\sin \left(\phi_{R \perp}+\phi_{S}\right) \sin \theta}$ implemented. On average the implementation of the $\phi_{h}$ dependence resulted in a distribution which has the same average value, but which is $10 \%$ broader, independent of the $M_{\pi \pi}, z$ or $x$ bin considered. Thus this effect is found to be small compared to the other effect of the acceptance described above.

\section{Author list}

The HERMES collaboration

A. Airapetian ${ }^{16}$, N. Akopov ${ }^{27}$, Z. Akopov ${ }^{27}$, A. Andrus ${ }^{15}$, E.C. Aschenauer ${ }^{7}$, W. Augustyniak ${ }^{26}$, R. Avakian ${ }^{27}$, A. Avetissian ${ }^{27}$, E. Avetissian ${ }^{11}$,

A. Bacchetta ${ }^{21}$, L. Barion ${ }^{10}$, S. Belostotski ${ }^{19}$, N. Bianchi ${ }^{11}$, H.P. Blok ${ }^{18,25}$, H. Böttcher ${ }^{7}$, C. Bonomo ${ }^{10}$, A. Borissov ${ }^{14}$, A. Borysenko ${ }^{11}$, A. Brüll ${ }^{\dagger}$, V. Bryzgalov ${ }^{20}$, M. Capiluppi ${ }^{10}$, G.P. Capitani ${ }^{11}$, E. Cisbani ${ }^{22}$, G. Ciullo ${ }^{10}$, M. Contalbrigo ${ }^{10}$, P.F. Dalpiaz ${ }^{10}$, W. Deconinck ${ }^{16}$, R. De Leo ${ }^{2}$, M. Demey ${ }^{18}$, L. De Nardo ${ }^{6,23}$, E. De Sanctis ${ }^{11}$, E. Devitsin ${ }^{17}$, M. Diefenthaler ${ }^{9}$, P. Di Nezza ${ }^{11}$, J. Dreschler ${ }^{18}$, M. Düren ${ }^{13}$, M. Ehrenfried ${ }^{9}$,

A. Elalaoui-Moulay ${ }^{1}$, G. Elbakian ${ }^{27}$, F. Ellinghaus ${ }^{5}$, U. Elschenbroich ${ }^{12}$,

R. Fabbri ${ }^{18}$, A. Fantoni ${ }^{11}$, L. Felawka ${ }^{23}$, S. Frullani ${ }^{22}$, A. Funel ${ }^{11}$,

G. Gapienko ${ }^{20}$, V. Gapienko ${ }^{20}$, F. Garibaldi ${ }^{22}$, G. Gavrilov ${ }^{6,19,23}$,

V. Gharibyan ${ }^{27}$, F. Giordano ${ }^{10}$, O. Grebeniouk ${ }^{10}$, I.M. Gregor ${ }^{7}$,

K. Griffioen ${ }^{18}$, H. Guler ${ }^{7}$, C. Hadjidakis ${ }^{11}$, M. Hartig ${ }^{6}$, D. Hasch ${ }^{11}$,

T. Hasegawa ${ }^{24}$, W.H.A. Hesselink ${ }^{18,25}$, G. Hill ${ }^{14}$, A. Hillenbrand ${ }^{9}$, M. Hoek ${ }^{13}$,

Y. Holler ${ }^{6}$, B. Hommez ${ }^{12}$, I. Hristova ${ }^{7}$, G. Iarygin ${ }^{8}$, Y. Imazu $^{24}$,

A. Ivanilov ${ }^{20}$, A. Izotov ${ }^{19}$, H.E. Jackson ${ }^{1}$, A. Jgoun ${ }^{19}$, R. Kaiser ${ }^{14}$, T. Keri ${ }^{13}$,

E. Kinney ${ }^{5}$, A. Kisselev ${ }^{5,19}$, T. Kobayashi ${ }^{24}$, M. Kopytin ${ }^{7}$, V. Korotkov ${ }^{20}$,

V. Kozlov ${ }^{17}$, B. Krauss ${ }^{9}$, P. Kravchenko ${ }^{19}$, V.G. Krivokhijine ${ }^{8}$, L. Lagamba $^{2}$, L. Lapikás ${ }^{18}$, P. Lenisa ${ }^{10}$, P. Liebing ${ }^{7}$, L.A. Linden-Levy ${ }^{15}$, W. Lorenzon ${ }^{16}$, S. $\mathbf{L u}{ }^{13}$, X. Lu ${ }^{24}$, B.-Q. $\mathbf{M a}^{3}$, B. Maiheu ${ }^{12}$, N.C.R. Makins ${ }^{15}$, Y. Mao ${ }^{3}$, B. Marianski ${ }^{26}$, H. Marukyan ${ }^{27}$, V. Mexner ${ }^{18}$, C.A. Miller ${ }^{23}$, Y. Miyachi ${ }^{24}$, V. Muccifora ${ }^{11}$, M. Murray ${ }^{14}$, A. Nagaitsev ${ }^{8}$, E. Nappi ${ }^{2}$, Y. Naryshkin ${ }^{19}$, M. Negodaev ${ }^{7}$, W.-D. Nowak ${ }^{7}$, A. Osborne ${ }^{14}$, L.L. Pappalardo ${ }^{10}$, R. Perez-Benito ${ }^{13}$, N. Pickert ${ }^{9}$, M. Raithel ${ }^{9}$, D. Reggiani ${ }^{9}$, P.E. Reimer ${ }^{1}$, A. Reischl ${ }^{18}$, A.R. Reolon ${ }^{11}$, C. Riedl ${ }^{9}$, K. Rith $^{9}$, S.E. Rock $^{6}$, G. Rosner ${ }^{14}$,

${ }^{\dagger}$ Present address: 36 Mizzen Circle, Hampton, Virginia 23664, USA 
A. $\operatorname{Rostomyan}^{6}$, L. Rubacek ${ }^{13}$, J. Rubin ${ }^{15}$, D. Ryckbosch ${ }^{12}$, Y. Salomatin ${ }^{20}$, I. Sanjiev ${ }^{1,19}$, A. Schäfer ${ }^{21}$, G. Schnell ${ }^{24}$, K.P. Schüler ${ }^{6}$, B. Seitz ${ }^{13}$, C. Shearer ${ }^{14}$, T.-A. Shibata ${ }^{24}$, V. Shutov $^{8}$, M. Stancari ${ }^{10}$, M. Statera ${ }^{10}$, E. Steffens ${ }^{9}$, J.J.M. Steijger ${ }^{18}$, H. Stenzel ${ }^{13}$, J. Stewart ${ }^{7}$, F. Stinzing ${ }^{9}$, J. Streit ${ }^{13}$, P. Tait ${ }^{9}$, S. Taroian ${ }^{27}$, B. Tchuiko ${ }^{20}$, A. Terkulov ${ }^{17}$, A. Trzcinski ${ }^{26}$, M. Tytgat ${ }^{12}$, A. Vandenbroucke ${ }^{12}$, P.B. van der Nat ${ }^{18}$, G. van der Steenhoven ${ }^{18}$, Y. van Haarlem ${ }^{12}$, C. van Hulse ${ }^{12}$,

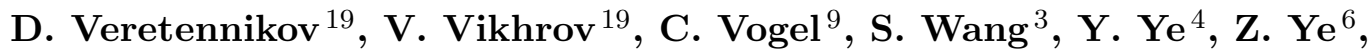
S. Yen ${ }^{23}$, D. Zeiler ${ }^{9}$, B. Zihlmann ${ }^{12}$, P. Zupranski ${ }^{26}$

1 Physics Division, Argonne National Laboratory, Argonne, Illinois 60439-4843, USA

2 Istituto Nazionale di Fisica Nucleare, Sezione di Bari, 70124 Bari, Italy

3 School of Physics, Peking University, Beijing 100871, China

4 Department of Modern Physics, University of Science and Technology of China, Hefei, Anhui 230026, China

5 Nuclear Physics Laboratory, University of Colorado, Boulder, Colorado 80309-0390, $U S A$

6 DESY, 22603 Hamburg, Germany

7 DESY, 15738 Zeuthen, Germany

8 Joint Institute for Nuclear Research, 141980 Dubna, Russia

9 Physikalisches Institut, Universität Erlangen-Nürnberg, 91058 Erlangen, Germany

10 Istituto Nazionale di Fisica Nucleare, Sezione di Ferrara and Dipartimento di Fisica, Università di Ferrara, 44100 Ferrara, Italy

11 Istituto Nazionale di Fisica Nucleare, Laboratori Nazionali di Frascati, 00044 Frascati, Italy

12 Department of Subatomic and Radiation Physics, University of Gent, 9000 Gent, Belgium

13 Physikalisches Institut, Universität Gießen, 35392 Gießen, Germany

14 Department of Physics and Astronomy, University of Glasgow, Glasgow G12 8QQ, United Kingdom

15 Department of Physics, University of Illinois, Urbana, Illinois 61801-3080, USA

16 Randall Laboratory of Physics, University of Michigan, Ann Arbor, Michigan 48109-1040, USA

17 Lebedev Physical Institute, 117924 Moscow, Russia

18 National Institute for Subatomic Physics (Nikhef), 1009 DB Amsterdam, The Netherlands

19 Petersburg Nuclear Physics Institute, St. Petersburg, Gatchina, 188350 Russia

20 Institute for High Energy Physics, Protvino, Moscow region, 142281 Russia

21 Institut für Theoretische Physik, Universität Regensburg, 93040 Regensburg, Germany

22 Istituto Nazionale di Fisica Nucleare, Sezione Roma 1, Gruppo Sanità and Physics Laboratory, Istituto Superiore di Sanità, 00161 Roma, Italy

23 TRIUMF, Vancouver, British Columbia V6T 2A3, Canada 
24 Department of Physics, Tokyo Institute of Technology, Tokyo 152, Japan

25 Department of Physics and Astronomy, Vrije Universiteit, $1081 \mathrm{HV}$ Amsterdam, The Netherlands

26 Andrzej Soltan Institute for Nuclear Studies, 00-689 Warsaw, Poland

27 Yerevan Physics Institute, 375036 Yerevan, Armenia

\section{References}

[1] V. Barone, A. Drago, and P. G. Ratcliffe Phys. Rept. 359 (2002) 1-168, hep-ph/0104283.

[2] A. De Rujula, J. M. Kaplan, and E. De Rafael Nucl. Phys. B35 (1971) 365-389.

[3] Hermes Collaboration, A. Airapetian et. al. Phys. Rev. Lett. 94 (2005) 012002, hep-ex/0408013.

[4] Compass Collaboration, V. Y. Alexakhin et. al. Phys. Rev. Lett. 94 (2005) 202002, hep-ex/0503002.

[5] Compass Collaboration, E. S. Ageev et. al. Nucl. Phys. B765 (2007) 31-70, hep-ex/0610068.

[6] J. C. Collins Nucl. Phys. B396 (1993) 161-182, hep-ph/9208213.

[7] D. W. Sivers Phys. Rev. D41 (1990) 83.

[8] X. Ji, J.-P. Ma, and F. Yuan Phys. Lett. B597 (2004) 299-308, hep-ph/0405085.

[9] J. C. Collins and A. Metz Phys. Rev. Lett. 93 (2004) 252001, hep-ph/0408249.

[10] M. Anselmino et. al. Phys. Rev. D75 (2007) 054032, hep-ph/0701006.

[11] A. V. Efremov, L. Mankiewicz, and N. A. Tornqvist Phys. Lett. B284 (1992) 394-400.

[12] J. C. Collins, S. F. Heppelmann, and G. A. Ladinsky Nucl. Phys. B420 (1994) 565-582, hep-ph/9305309.

[13] K. Konishi, A. Ukawa, and G. Veneziano Phys. Lett. B78 (1978) 243.

[14] J. C. Collins and G. A. Ladinsky hep-ph/9411444.

[15] R. L. Jaffe, X. Jin, and J. Tang Phys. Rev. Lett. 80 (1998) 1166-1169, hep-ph/9709322.

[16] X. Artru and J. C. Collins Z. Phys. C69 (1996) 277-286, hep-ph/9504220].

[17] M. Stratmann and W. Vogelsang Phys. Lett. B295 (1992) 277-282.

[18] D. Boer, R. Jakob, and M. Radici Phys. Rev. D67 (2003) 094003, hep-ph/0302232.

[19] A. Bianconi, S. Boffi, R. Jakob, and M. Radici Phys. Rev. D62 (2000) 034008, hep-ph/9907475.

[20] A. Bacchetta and M. Radici Phys. Rev. D69 (2004) 074026, hep-ph/0311173.

[21] A. V. Efremov and O. V. Teryaev Sov. J. Nucl. Phys. 36 (1982) 140.

[22] X. Ji Phys. Rev. D49 (1994) 114-124, hep-ph/9307235.

[23] M. Anselmino, M. Bertini, F. Caruso, F. Murgia, and P. Quintairos Eur. Phys. J. C11 (1999) 529-537, hep-ph/9904205.

[24] A. Bacchetta and P. J. Mulders Phys. Rev. D62 (2000) 114004, hep-ph/0007120. 
[25] I. Cohen et. al. Phys. Rev. D25 (1982) 634.

[26] European Muon Collaboration, J. J. Aubert et. al. Phys. Lett. B133 (1983) 370.

[27] European Muon Collaboration, M. Arneodo et. al. Z. Phys. C33 (1986) 167.

[28] Hermes Collaboration, A. Airapetian et. al. Phys. Rev. Lett. 96 (2006) 162301, hep-ex/0510030.

[29] A. Majumder and X.-N. Wang Phys. Rev. D70 (2004) 014007, hep-ph/0402245.

[30] Delphi Collaboration, P. Abreu et. al. Phys. Lett. B406 (1997) 271-286.

[31] Opal Collaboration, G. Abbiendi et. al. Eur. Phys. J. C16 (2000) 61-70, hep-ex/9906043.

[32] Q.-h. Xu, C.-x. Liu, and Z.-t. Liang Phys. Rev. D63 (2001) 111301, hep-ph/0103267.

[33] Q.-h. Xu and Z.-t. Liang Phys. Rev. D67 (2003) 114013, hep-ph/0304125.

[34] Sld Collaboration, K. Abe et. al. Phys. Rev. Lett. 74 (1995) 1512-1516, hep-ex/9501006.

[35] D. de Florian and L. Vanni Phys. Lett. B578 (2004) 139-149, hep-ph/0310196.

[36] F. A. Ceccopieri, M. Radici, and A. Bacchetta Phys. Lett. B650 (2007) 81-89, hep-ph/0703265.

[37] M. Radici, R. Jakob, and A. Bianconi Phys. Rev. D65 (2002) 074031, hep-ph/0110252.

[38] A. Bacchetta and M. Radici Phys. Rev. D74 (2006) 114007, hep-ph/0608037.

[39] A. Nass et. al. Nucl. Instrum. Meth. A505 (2003) 633-644.

[40] Hermes Collaboration, A. Airapetian et. al. Nucl. Instrum. Meth. A540 (2005) 68-101, physics/0408137.

[41] Hermes Collaboration, K. Ackerstaff et. al. Nucl. Instrum. Meth. A417 (1998) 230-265, hep-ex/9806008.

[42] N. Akopov et. al. Nucl. Instrum. Meth. A479 (2002) 511-530, physics/0104033.

[43] A. Bacchetta and M. Radici Phys. Rev. D67 (2003) 094002, hep-ph/0212300.

[44] A. Bacchetta, U. D'Alesio, M. Diehl, and C. A. Miller Phys. Rev. D70 (2004) 117504, hep-ph/0410050.

[45] T. Sjöstrand, L. Lönnblad, S. Mrenna, and P. Skands hep-ph/0308153.

[46] P. Liebing. PhD thesis, 2004. DESY-THESIS-2004-036.

[47] M. Diehl and S. Sapeta Eur. Phys. J. C41 (2005) 515-533, hep-ph/0503023.

[48] Hermes Collaboration, P. B. van der Nat and K. Griffioen hep-ex/0501009.

[49] L. W. Whitlow, S. Rock, A. Bodek, E. M. Riordan, and S. Dasu Phys. Lett. B250 (1990) 193-198.

[50] J. She, Y. Huang, V. Barone, and B.-Q. Ma 0711.0817.

[51] M. Glück, E. Reya, and A. Vogt Eur. Phys. J. C5 (1998) 461-470, hep-ph/9806404.

[52] P. Schweitzer et. al. Phys. Rev. D64 (2001) 034013, hep-ph/0101300.

[53] P. B. van der Nat. PhD thesis, 2007. 